\title{
Extractos hidro-etanólicos de plantas comestibles como alternativa para controlar bacterias patógenas, parásitos e insectos en la industria pecuaria
}

\author{
Hydro-ethanolic extracts of edible plants as an alternative to control pathogenic bacteria, parasites and \\ insects in the livestock industry
}

\begin{abstract}
Hernán Celaya-Michel', Jesús Anaya-Islas' ${ }^{1}$, Miguel Ángel Barrera-Silva', Susana Marlene Barrales-Heredia', Manuel Nieblas-López' ${ }^{1}$, Reyna Fabiola Osuna-Chávez', Cristina Ibarra-Zazueta', Guadalupe López-Robles', Priscilia Yazmín Heredia-Castro², Jesús Sosa-Castañeda ${ }^{1 *}$

Departamento de Agricultura y Ganadería, Universidad de Sonora. Carretera a Bahía de Kino Km. 21. Apartado Postal 305. Hermosillo, Sonora, México.

2 Laboratorio de Calidad, Autenticidad y Trazabilidad de los Alimentos. Centro de Investigación en Alimentación y Desarrollo, A.C. (CIAD). Carretera a La Victoria Km 0.6, Apartado 1735, Hermosillo, Sonora, 83304. México.
\end{abstract}

\section{RESUMEN}

En la industria pecuaria es importante mantener al ganado en buen estado de salud para alcanzar niveles adecuados de producción. En la actualidad, el uso de antibióticos para el control de infecciones es limitado, debido a la resistencia de los microorganismos a estos fármacos. Por otro lado, los insecticidas y repelentes de origen sintéticos contienen químicos tóxicos para los animales, el humano y el medio ambiente. Una alternativa es utilizar compuestos químicos naturales derivados de plantas. El objetivo fue evaluar la actividad antimicrobiana, insecticida y repelente de extractos hidro-etanólicos de plantas. En este estudio, se utilizaron 12 extractos de plantas contra 5 diferentes bacterias y 8 especies de insectos. Los resultados mostraron que el extracto de Eucalyptus globulus y la mezcla de extractos presentaron actividad antimicrobiana contra Escherichia coli, Listeria monocytogenes, Salmonella typhimurium, Vibrio cholerae y Staphylococcus aureus $(\mathrm{P}<0.05)$. Por otro lado, los extractos de E. globulus y Azadirachta indica mostraron actividad insecticida contra todos los insectos $(P<0.05)$; mientras que, la mezcla de los extractos mostró actividad repelente contra todos los insectos utilizados $(\mathrm{P}<0.05)$. Por lo anterior, los extractos hidro-etanólicos de plantas mostraron potencial para controlar bacterias patógenas e insectos indeseables en la industria pecuaria.

Palabras clave: Ganado, antimicrobiano, insecticida, repelente, extractos hidro-etanólicos.

\section{ABSTRACT}

In the livestock industry, it is important to keep livestock in good health to reach adequate levels of production. Currently, the use of antibiotics for the control of infections is limited, due to the resistance of microorganisms to these drugs. On the other hand, the insecticides and repellents of synthetic origin contain toxic chemicals for animals, humans and the environment. An alternative is to use natural chemical compounds derived from plants. The objective was to evaluate the antimicrobial, insecticide and repellent activity of hydro-ethanolic extracts of plants. In this study, we used 12 plant extracts against five different bacteria and eight insect species. The results showed that the extract of Eucalyptus globulus and the mixture of extracts showed antimicrobial activity against Escherichia coli, Listeria monocytogenes, Salmonella typhimurium, Vibrio cholerae and Staphylococcus aureus $(\mathrm{P}<0.05)$. On the other hand, extracts of $E$. globulus and Azadirachta indica showed insecticidal activity against all insects $(P<0.05)$; while the mixture of the extracts showed repellent activity against all insects used $(\mathrm{P}<0.05)$. Therefore, the hydro-ethanolic extracts of plants showed potential to control pathogenic bacteria and undesirable insects in the livestock industry.

Keywords: Cattle, antimicrobial, insecticide, repellent, hydro-ethanolic extracts.

\section{INTRODUCCIÓN}

La contaminación microbiológica y las enfermedades causadas por bacterias patógenas y parásitos externos son las principales causas de pérdidas económicas en la industria ganadera (Rodríguez-Vivas et al., 2017). Durante décadas, diversas investigaciones se han enfocado en estrategias para mejorar la producción animal. Una de las estrategias a seguir para reducir el crecimiento microbiano son los antibióticos, sin embargo, su uso excesivo ha ocasionado que las bacterias se adapten y desarrollen resistencia a estos fármacos, lo que ha generado mayor inversión económica para su control (Abdullah et al., 2011; Abraham y Pal, 2014). Además, se ha demostrado que el uso de antibióticos en el ganado destinado para la alimentación humana, puede contribuir a aumentar la resistencia de las bacterias a los antibióticos utilizados para tratar enfermedades en el humano (Landers et al., 2012; Barriere, 2015).

Staphylococcus aureus es un microorganismo patógeno que se puede encontrar en las industrias ganaderas; este patógeno puede infectar la glándula mamaria (mastitis) y generar pérdidas de leche que pueden alcanzar hasta el 70\% por vaca por ciclo de lactación (Shaheen et al., 2016); mientras que, E. coli, S. typhimurium, V. cholerae y L. monocytogenes

\footnotetext{
*Autor para correspondencia: Jesús Sosa-Castañeda

Correo electrónico: jesus.sosa@unison.mx
}

Volumen XXI, Número 2

Recibido: 9 de abril de 2018 
son patógenos que causan infecciones gastrointestinales y pueden disminuir la producción de leche y carne en el ganado (Abakpa et al., 2013; Manyi-Loh et al., 2016).

Por otro lado, las moscas, mosquitos y garrapatas son vectores importantes de enfermedades para el ganado, ya que son insectos consumidores de sangre y pueden alimentarse de animales enfermos e infectar animales sanos causándoles serios problemas de salud. Otros insectos, tales como las cucarachas, sirven como vehículos transportadores de microorganismos, ya que usualmente habitan cualquier sitio, incluyendo lugares contaminados por animales enfermos. Las termitas y las hormigas fabrican sus nidos y establecen sus colonias frecuentemente en los corrales, comederos, cercos, almacenes, o cualquier otro sitio, causando daño en la infraestructura de las instalaciones pecuarias y en cualquier otro utensilio o herramienta fabricada a base de madera, lo que implica una renovación constante de material e infraestructura de la empresa. Por lo anterior, el uso de insecticidas y repelentes de insectos es una práctica común en la industria pecuaria. Sin embargo, los insecticidas comúnmente utilizados están elaborados a base de químicos sintéticos, por ejemplo, algunos insecticidas son considerados como fármacos y pueden ser inyectados vía subcutánea, como es el caso de las lactonas macrocíclicas (ivermectina, moxidectina, doramectina) y las fenilpirazolonas (fipronil), mientras que, otros insecticidas pueden ser aplicados por inmersión, derrame dorsal o aspersión, como es el caso de los organofosforados (coumafos, clorpirifos, clorfenvinfos) y los piretroides (cipermetrina, deltametrina, flumetrina) (Martínez y Cruz, 2009; Rodríguez-Vivas et al., 2014). La desventaja del uso de estos compuestos químicos es que pueden acumularse dentro del organismo de los animales y contaminar el producto final (carne y leche), o bien, ocasionar daño en la salud de los animales por su uso excesivo, lo que puede llegar a generar pérdidas económicas importantes. Además, el uso inadecuado de los insecticidas y repelentes de insectos puede afectar la salud del humano y del medio ambiente (del Puerto Rodrí- guez et al., 2014; Pimentel y Burgess, 2014). Por lo anterior, algunas investigaciones se han enfocado en la extracción de compuestos químicos de plantas para ser utilizados como agentes antimicrobianos (Silva y Fernandes, 2010; Elisha et al., 2017), insecticidas (Borges et al., 2011; Volpato et al., 2016) y repelentes de insectos (Maia y Moore, 2011; Castillo et al., 2017). Sin embargo, son poco los estudios que evalúan la actividad antimicrobiana, insecticida y repelente de plantas comestibles y lo enfocan como una alternativa natural para la industria pecuaria. Además, la actividad biológica de cada planta puede variar, por lo que la búsqueda de otras especies de plantas para este fin es de gran importancia. Entre las ventajas de los compuestos naturales, se encuentran su fácil degradación, menor toxicidad y no contaminan (Celis et al., 2008; George et al., 2014).

Tomando en cuenta lo anterior, los extractos de plantas pueden representar una opción viable para este fin. Además, el mercado de alimentos orgánicos, hoy en día ha ido en aumento debido a que el consumidor prefiere los productos derivados de origen animal que no hayan sido tratados con químicos sintéticos, lo que abre la posibilidad de incursionar en este nuevo mercado. El objetivo de esta investigación fue evaluar la capacidad de los extractos hidro-etanólicos de plantas comestibles para eliminar bacterias patógenas, parásitos externos e insectos comunes en la industria pecuaria.

\section{MATERIALES Y MÉTODOS}

\section{Preparación de los extractos hidro-etanólicos}

Los extractos se obtuvieron a partir de 12 plantas comestibles (Tabla 1), donde cada planta fue deshidratada a 34 ${ }^{\circ} \mathrm{C}$ en una estufa de aire caliente (Thelco, Precision Science, modelo 28, USA) y pulverizada en un molino (Pulvex Mini $100, M x$ ) hasta obtener un tamaño de partícula de 100 micras. Posteriormente, se colocaron $50 \mathrm{~g}$ de material seco y pulverizado y se agregaron $200 \mathrm{~mL}$ de una solución preparada con $70 \%$ de etanol absoluto (Sigma-Aldrich, St. Louis MO) y 30 $\%$ de agua destilada en un frasco de vidrio herméticamente

Tabla 1. Rendimiento de los extractos hidro-etanólicos por cada $50 \mathrm{~g}$ de materia seca.

Table 1. Yield of hydro-ethanolic extracts for each $50 \mathrm{~g}$ of dry matter.

\begin{tabular}{lcccc}
\hline Especie de planta & Familia & Nombre común & Parte de la planta & Rendimiento (\%) \\
\hline Azadirachta indica & Meliaceae & Neem & Hojas & 5.5 \\
\hline Cinnamomum verum & Lauraceae & Canela & Corteza & 4.8 \\
\hline Eucalyptus globulus & Myrtaseae & Eucalipto & Hojas & 5.7 \\
Laurus nobilis & Lauraceae & Laurel & Hojas & 5.2 \\
\hline Mentha spicata & Lamiaceae & Hierbabuena & Hojas & 5.5 \\
\hline Mentha spp & Lamiaceae & Menta & Hojas & 5.8 \\
Moringa oleifera & Moringaceae & Moringa & Hojas & 5.2 \\
Origanum vulgare & Lamiaceae & Orégano & Hojas & 5.6 \\
\hline Rosmarinus officinalis & Lamiaceae & Romero & Hojas & 5.5 \\
\hline Salvia hispánica & Lamiaceae & Chía & Semillas & 7.5 \\
Syzygium aromaticum & Myrtaseae & Clavo & Botón floral & 6.3 \\
Thymus vulgaris & Lamiaceae & Tomillo & Hojas & 7.2 \\
\hline
\end{tabular}

* Rendimiento $=\mathrm{g} / 100 \mathrm{~mL}$. 
sellado (por cada planta). Posteriormente, se almacenaron por 48 horas en la obscuridad a $25^{\circ} \mathrm{C}$. Después, los extractos fueron filtrados con papel filtro Whatman No. 41 y el material vegetal fue deshidratado nuevamente. La diferencia de peso del material vegetal antes y después de su almacenamiento fue considerado como la cantidad de compuestos químicos solubles extraídos de las plantas comestibles (Pulido y Cruz, 2013; Mostafa et al., 2017). Los extractos fueron ajustados a una concentración de $5 \mathrm{~g} / 100 \mathrm{~mL}$ en etanol al $70 \%$, mientras que la mezcla de extractos hidro-etanólicos se preparó en proporción 1:1 con cada uno de los extractos y se ajustó a una concentración final de $5 \mathrm{~g} / 100 \mathrm{~mL}$ en etanol al $70 \%$. Finalmente, los extractos hidro-etanólicos fueron almacenados en la obscuridad a $25^{\circ} \mathrm{C}$ hasta su utilización.

\section{Actividad antimicrobiana}

La actividad antimicrobiana de los extractos se evaluó contra bacterias Gram positivas (L. monocytogenes ATCC 19115 y S. aureus ATCC 25923) y bacterias Gram negativas (E. coli ATCC 25922, S. typhimurium ATCC 14028 y V. cholerae ATCC 14033), las cuales se obtuvieron de la colección de cepas del Laboratorio de Microbiología del Departamento de Ciencias Químico Biológicas de la Universidad de Sonora. Las cepas fueron propagadas en el medio de cultivo caldo MueIler Hinton (Sigma-Aldrich, St. Louis MO). Posteriormente, las bacterias fueron cosechadas en base a su curva de crecimiento en los tiempos de incubación donde se encontraban a una concentración de $10^{7} \mathrm{UFC} / \mathrm{mL}$. Después, se prepararon 2 placas con agar Mueller Hinton (Sigma-Aldrich, St. Louis MO) para cada una de las cepas y se colocaron 4 discos estériles de papel filtro Whatman No. 41 de $6 \mathrm{~mm}$ de diámetro en cada placa. Cada uno de los discos fueron adicionados con $20 \mu \mathrm{L}$ de cada extracto hidro-etanólico y con la mezcla de extractos. Finalmente, las placas fueron incubadas a $37^{\circ} \mathrm{C}$ por $24 \mathrm{~h}$. Los halos mayores o iguales a $8 \mathrm{~mm}$ fueron considerados como inhibición de acuerdo a los criterios utilizados por Alzamora et al. (2001).

\section{Actividad insecticida in vitro}

La actividad insecticida se determinó siguiendo la metodología reportada por Gemeda et al. (2014) y Simonetti y Christ (2017), con modificaciones. Para lo cual, se utilizaron 8 especies diferentes de insectos (Tabla 2 ) y se colocaron 25 insectos de la misma especie en cajas de plástico transparente de $20 \times 20 \times 10 \mathrm{~cm}$. Posteriormente, se colocó papel filtro Whatman No. 1 dentro de las cajas y cada insecto fue expuesto a $1 \mathrm{~mL}$ de cada uno de los extractos hidro-etanólicos y con la mezcla de extractos utilizando un atomizador (contacto directo). El tiempo en que el insecto dejó de moverse fue considerado como el tiempo efectivo de muerte.

\section{Actividad repelente in vitro}

La actividad repelente se determinó siguiendo la metodología descrita por Jaramillo-Colorado et al. (2015), con modificaciones. Para lo cual, se utilizaron cajas de plástico transparente $(40 \times 30 \times 20 \mathrm{~cm})$, colocando en el extremo iz-
Tabla 2. Especies de insectos utilizados en los bioensayos. Table 2. Insect species used in the bioassays.

\begin{tabular}{lcc}
\hline Especie de insecto & Familia & Nombre común \\
\hline Aedes aegypti & Culicidae & Mosquito \\
\hline Camponotus spp. & Formicidae & Hormiga carpintera \\
\hline Cryptotermes brevis & Kalotermitidae & Termita \\
\hline Periplaneta americana & Blattidae & Cucaracha \\
Pholcus phalangioides & Pholcidae & Araña patona \\
Rhipicephalus microplus & Ixodidae & Garrapata \\
Solenopsis spp. & Formicidae & Hormiga de fuego \\
Stomoxys calcitrans & Muscidae & Mosca del establo \\
\hline
\end{tabular}

quierdo de éstas papel filtro Whatman No. 1 adicionado con los extractos hidro-etanólicos y con la mezcla de extractos. Mientras que, en el extremo derecho de la caja se colocó papel filtro con alcohol al $70 \%$. Una vez evaporado el alcohol, se colocaron 25 insectos en la parte central de la caja y se esperó un tiempo de $5 \mathrm{~min}$ para que los insectos tomaran su ubicación dentro de la caja. Finalmente, el tiempo de permanencia de los insectos en la parte derecha de la caja fue considerado como el tiempo efectivo de repelencia.

\section{Análisis estadístico}

Se utilizó un diseño experimental completamente al azar con un ANOVA al 95\% de confianza. La prueba de comparación de medias se realizó por la prueba de Tukey y Kramer a un nivel de significancia de $0.5 \%$. Se utilizaron dos repeticiones por tratamiento para el análisis microbiológico y 25 repeticiones por tratamiento para los análisis de actividad insecticida y repelente. El paquete estadístico utilizado fue el software NCSS 2012 versión 12.0.1.

\section{RESULTADOS Y DISCUSIÓN}

Durante miles de años las plantas han evolucionado para protegerse contra los ataques de los animales herbívoros y los microorganismos patógenos a través de la producción de compuestos fitoquímicos. Estos compuestos suelen encontrarse en el tejido vegetal o en la savia que ellas mismas elaboran a través de la absorción de nutrientes; y la producción y secreción de metabolitos (Diaz et al., 2017). Por lo anterior, es común que el rendimiento en cuanto a la extracción de compuestos fitoquímicos derivados de las plantas varíe de una planta a otra (Tabla 1). Recientemente Mostafa et al. (2017), han reportado rendimientos de 6.54 $\%$ para Thymus vulgaris y $4.38 \%$ para Syzygium aromaticum, lo que representa un rendimiento menor de $18.87 \%$ y 5.88 $\%$ en comparación con lo encontrado en este estudio para estas mismas especies de plantas. Otros factores adicionales que pudieran afectar el rendimiento de la extracción de los compuestos fitoquímicos de las plantas podría ser el tipo de solvente utilizado en la extracción de los compuestos, la cantidad de compuestos solubles presentes en cada especie de planta y/o la calidad del material vegetal utilizado (Azwanida, 2015). 
Algunas investigaciones han asociados la actividad antimicrobiana de los extractos de plantas a los compuestos fenólicos, alcaloides, lectinas, poliacetilenos, ácido gálico, ácido eicosanóico, miristicina, berbelina, friedelina, entre otros; y se menciona que estos compuestos pueden variar entre las especies de plantas, e incluso entre la misma variedad (Silva y Fernandes, 2010; Anyanwu y Okoye, 2017). En este estudio los extractos hidro-etanólicos de las plantas comestibles fueron capaces de eliminar cuando menos a una bacteria patógena $(P<0.05)$, siendo el extracto-hidroetanólico de E. globulus el más eficiente $(P<0.05)$ (Tabla 3$)$. Asimismo, la mezcla de extractos hidro-etanólicos mostró ser más eficiente con respecto a la actividad antimicrobiana de los extractos evaluada de manera individual $(\mathrm{P}<0.05)$.

Otros estudios, han reportado que algunos extractos de plantas han mostrado actividad antimicrobiana contra bacterias patógenas (Munuswamy et al., 2013). Al-Mariri y Safi, (2014) reportaron que el extracto etanólico de Laurus nobilis mostró actividad antimicrobiana contra E. coli, mientras que, los aceites de Origanos syriacum, Thymus syriacus, $S$. aromaticum, y Cinnamomum zeylanicum mostraron actividad antimicrobiana contra esa misma cepa. Por otro lado, los autores reportaron que Eucalyptus camaldulensis no presentó actividad antimicrobiana contra E. coli, mientras que, en este estudio el extracto hidro-etanólico de E. globulus sí presentó actividad antimicrobiana contra ese patógeno. Lo anterior sugiere que la actividad antimicrobiana entre especies de plantas de la misma familia puede presentar variación en su actividad biológica debido a la presencia y cantidad de los diferentes compuestos químicos de sus hojas, tales como, globulol, 1-8 cineol, Trans-Pinocarveol, entre otros (Sebei et al., 2015). Otro estudio mostró que el extracto metanólico de Azadirachta indica presentó actividad antimicrobiana contra E. coli y Salmonella. En nuestro estudio, A. indica no presentó actividad antimicrobiana contra esos patógenos, lo que sugiere que el solvente utilizado posiblemente influyó en la actividad biológica debido a la naturaleza de los compuestos químicos solubles (Panchal et al., 2013). También se ha reportado que el extracto acuoso de S. aromaticum presentó actividad antimicrobiana contra S. aureus. Aunque el extracto de $S$. aromaticum fue distinto al utilizado en este estudio, la actividad antimicrobiana fue similar para ese patógeno cuando se utilizó la misma especie de planta (Ajiboye et al., 2016). Se ha sugerido que el mecanismo de acción antimicrobiano de los compuestos de las plantas varía de acuerdo al tipo de moléculas presentes en ellas. Por ejemplo, los compuestos fenólicos pueden interactuar con algunas enzimas microbianas, tales como las ATPasas e inhibir su acción causando la muerte de la bacteria (Abachi et al., 2016); los terpenos y aceites esenciales actúan rompiendo la membrana celular a través de su interacción con los compuestos lipofílicos; y los alcaloides suelen unirse al ADN de las bacterias y dañar el material genético causándoles la muerte (Andrade-Bustamante, 2017).

Algunos autores han asociado a los aldehídos, terpenoides, compuestos fenólicos, furanos, alcaloides, entre otros, con la actividad insecticida de los extractos de plantas (Vázquez-Luna et al., 2007). En este estudio, los resultados de la actividad insecticida mostraron que los extractos hidroetanólicos de E. globulus y A. indica presentaron actividad contra todos los insectos utilizados, siendo estos más eficientes contra Aedes aegypti y menos eficientes contra Camponotus spp. $(\mathrm{P}<0.05)($ Tabla 4$)$. Por otra parte, la mezcla de extractos hidro-etanólicos fue más eficiente con respecto a todos los extractos evaluados de manera individual $(\mathrm{P}<0.05)$. Por ejemplo, los resultados muestran que la mezcla de extractos hidro-etanólicos aumentó la actividad insecticida en un $\mathbf{9 5 . 0 5 \%}$ en Camponotus spp., $75.87 \%$ en Periplaneta

Tabla 3. Actividad antimicrobiana de los extractos hidro-etanólicos de plantas comestibles.

Table 3. Antimicrobial activity of the hydro-ethanolic extracts of edible plants.

\begin{tabular}{|c|c|c|c|c|c|}
\hline \multirow[b]{2}{*}{ Especie de planta } & \multicolumn{5}{|c|}{ Halos de inhibición de las bacterias patógenas ( $\mathrm{mm}$ ) } \\
\hline & Escherichia coli & Listeria monocytogenes & Salmonella typhimurium & Staphylococcus aerus & Vibrio cholerae \\
\hline Azadirachta indica & - & - & - & - & $16.33 \pm 1.53^{\mathrm{d}}$ \\
\hline Cinnamomum verum & $10.33 \pm 0.58^{b}$ & $11.36 \pm 0.56^{b}$ & - & - & $19.33 \pm 1.53^{e}$ \\
\hline Eucalyptus globulus & $11.67 \pm 0.57^{c}$ & $10.67 \pm 0.55^{\mathrm{a}}$ & $10.00 \pm 1.00^{\mathrm{b}}$ & $10.00 \pm 1.00^{\mathrm{a}}$ & $13.67 \pm 1.15^{c}$ \\
\hline Laurus nobilis & $12.8 \pm 0.58^{d}$ & $11.33 \pm 0.58^{b}$ & - & - & $25.33 \pm 0.58^{h}$ \\
\hline Mentha spicata & $11.67 \pm 0.56^{c}$ & - & - & $24.00 \pm 1.00^{c}$ & $15.67 \pm 0.57^{d}$ \\
\hline Mentha spp & - & - & $7.67 \pm 0.58^{\mathrm{a}}$ & - & $12.33 \pm 0.58^{b}$ \\
\hline Moringa oleífera & $9.67 \pm 0.58^{\mathrm{a}}$ & - & - & - & $13.67 \pm 0.58^{c}$ \\
\hline Origanum vulgare & - & - & - & $9.67 \pm 0.56^{a}$ & $9.33 \pm 0.58^{\mathrm{a}}$ \\
\hline Rosmarinus officinalis & - & $14.67 \pm 0.58^{d}$ & - & - & $21.00 \pm 1.00^{f}$ \\
\hline Salvia hispánica & $12.67 \pm 1.15^{d}$ & $12.33 \pm 0.58^{c}$ & $20.33 \pm 0.57^{e}$ & - & $12.67 \pm 0.56^{b}$ \\
\hline Syzygium aromaticum & $21.00 \pm 1.00^{f}$ & $18.00 \pm 1.73^{e}$ & - & $24.67 \pm 0.58^{c}$ & $23.00 \pm 1.00^{\mathrm{g}}$ \\
\hline Thymus vulgaris & $13.00 \pm 0.00^{e}$ & $14.67 \pm 0.54^{d}$ & $12.00 \pm 1.00^{c}$ & - & $21.00 \pm 1.00^{f}$ \\
\hline MEZCLA & $13.33 \pm 0.58^{\mathrm{e}}$ & $21.00 \pm 1.00^{f}$ & $14.33 \pm 0.58^{d}$ & $11.67 \pm 0.58^{b}$ & $20.00 \pm 1.00^{\mathrm{e}}$ \\
\hline
\end{tabular}

*Diferente literal indica diferencia significativa entre columnas $(P<0.05)$. Los resultados están expresados en milímetros. 
Tabla 4. Actividad insecticida de los extractos hidro-etanólicos de plantas comestibles. Table 4. Insecticidal activity of the hydro-ethanolic extracts of edible plants.

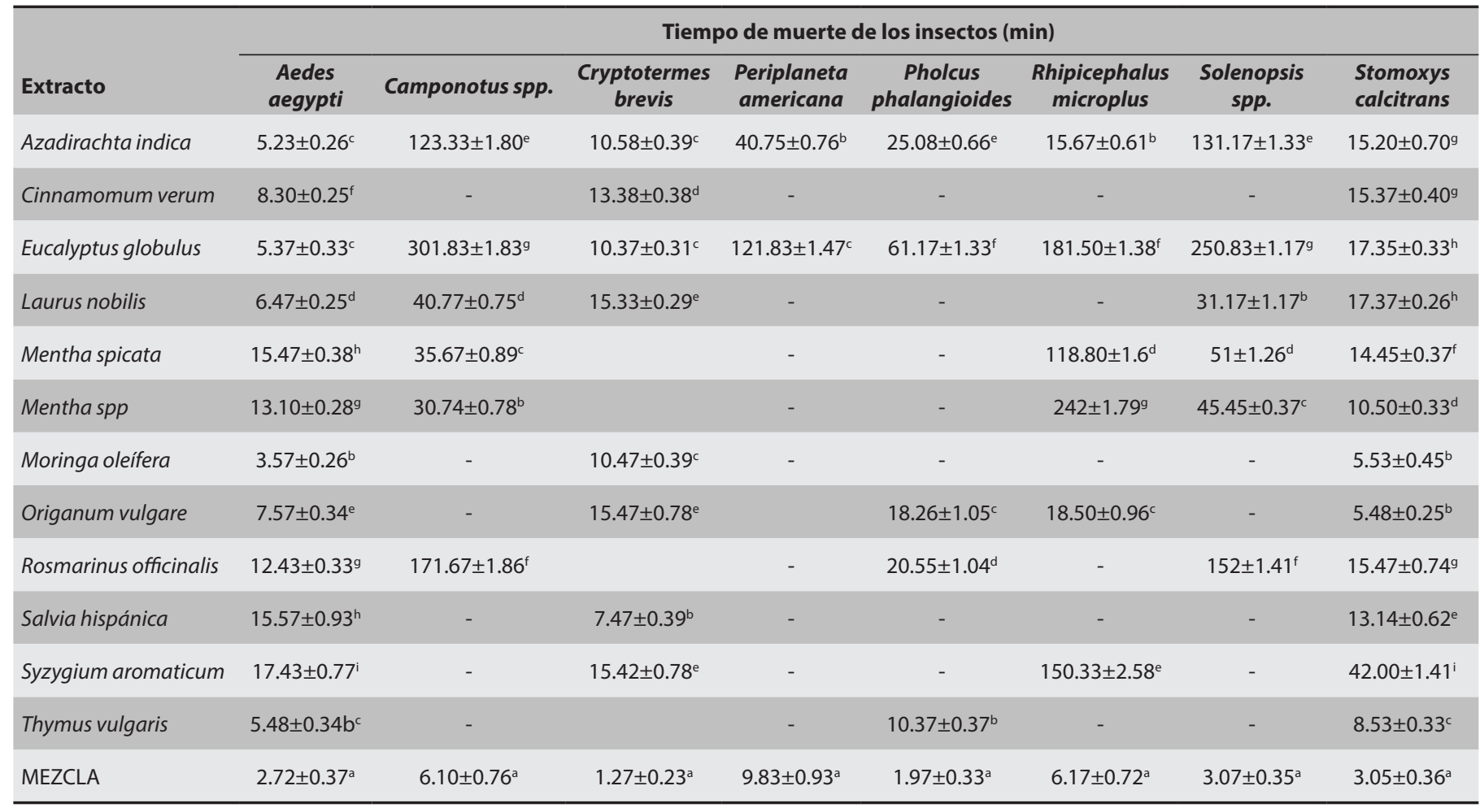

* Diferente literal indica diferencia significativa entre columnas $(P<0.05)$. Los resultados están expresados en minutos.

americana, $92.14 \%$ en Pholcus phalangioides y 79.93, 47.99, $97.65,87.99$ y $60.62 \%$ en Stomoxys calcitrans, A. aegypti, Solenopsis spp., Cryptotermes brevis y Rhipicephalus microplus, respectivamente; con respecto a la actividad insecticida de $A$. indica. Otros estudios también han reportado que los extractos de plantas presentan actividad insecticida contra diferentes especies de insectos (Zoubiri y Baaliouamer, 2014). En un estudio se reportó que $A$. indica logro eliminar a Reticulitermes flavipes (Tindo et al., 2012), sin embargo, en nuestro estudio, el extracto hidro-etanólico de esa misma planta fue más eficiente para eliminar a C. brevis.

Estos resultados pueden ser atribuidos a que ambos insectos son especies diferentes de termitas, lo que sugiere que los compuestos químicos de una misma especie de planta no garantizan su efectividad contra insectos de la misma familia. Por otro lado, se ha reportado que el aceite de Cinnamomum verum fue eficaz para eliminar a larvas $y$ hembras de R. microplus (Monteiro et al., 2017). Lo anterior difiere con los resultados encontrados en este estudio, ya que $C$. verum no presentó actividad insecticida contra esa especie de insecto. También fue reportado que el extracto acuoso de Moringa oleifera mostró actividad larvicida contra Culex quinquefasciatus, siendo eficaz para el control de mosquitos (Ashfaq y Ashfaq et al., 2012) y el aceite de E. globulus mostró actividad insecticida contra Musca domestica (Kumar et al., 2012).

En este estudio, también se presentó actividad insecticida contra mosquitos y moscas utilizando esa misma especie de planta, aun cuando la especie de mosquito y mosca utilizada en este estudio fue diferente a la reportada por Kumar et al. (2012). Hasta el momento, no se conocen todos los mecanismos de acción insecticida de los compuestos químicos de las plantas, sin embargo, se ha sugerido que los flavonoides forman complejos enzimáticos que modifican el metabolismo del insecto causándoles la muerte, o bien, pueden influir en su conducta, crecimiento y desarrollo a través de la síntesis de quinonas, producto de la oxidación de estos compuestos. Por otro lado, se ha sugerido que algunos péptidos cortos pueden inhibir la acción de las proteasas a nivel digestivo y causar la muerte del insecto (War et al., 2012).

Otros estudios han reportado que los extractos de plantas presentan actividad repelente contra diferentes especies de insectos (Niroumand et al., 2016). En este estudio, la actividad repelente de los extractos hidro-etanólicos mostraron que todos los extractos presentaron actividad repelente contra todos los insectos probados $(P<0.05)$ (Tabla 5). El extracto hidro-etanólico de $A$. indica fue el más efectivo para repeler a los insectos $(P>0.05)$, sin embargo, la mezcla de extractos hidro-etanólicos aumentó la actividad repelente en un $1.96 \%$ en Camponotus spp., $6.0 \%$ en P. americana, $2.88 \%$ en $P$. phalangioides, $1.12,3.40$ y $1.4 \%$ en $S$. calcitrans, C. brevis y R. microplus, respectivamente. Mandal (2011) reportaron que los aceites de Eucalyptus y A. indica mostraron actividad repelente contra C. quinquefasciatus hasta por $280 \mathrm{~min}$. En nuestro estudio encontramos que la actividad repelente de E. golobulus y A. indica para A. aegypti se mantuvo aproximadamente por $192 \mathrm{~min}$. La diferencia en el tiempo efectivo de 
Tabla 5. Actividad repelente de los extractos hidro-etanólicos de plantas comestibles. Table 5. Repellent activity of the hydro-ethanolic extracts of edible plants.

\begin{tabular}{|c|c|c|c|c|c|c|c|c|}
\hline \multirow[b]{2}{*}{ Extracto } & \multicolumn{8}{|c|}{ Tiempo de repelencia en los insectos (horas) } \\
\hline & $\begin{array}{l}\text { Aedes } \\
\text { aegypti }\end{array}$ & Camponotus spp. & $\begin{array}{c}\text { Cryptotermes } \\
\text { brevis }\end{array}$ & $\begin{array}{c}\text { Periplaneta } \\
\text { americana }\end{array}$ & $\begin{array}{c}\text { Pholcus } \\
\text { phalangioides }\end{array}$ & $\begin{array}{c}\text { Rhipicephalus } \\
\text { microplus }\end{array}$ & $\begin{array}{c}\text { Solenopsis } \\
\text { spp. }\end{array}$ & $\begin{array}{l}\text { Stomoxys } \\
\text { calcitrans }\end{array}$ \\
\hline Azadirachta indica & $3.16 \pm 0.13^{9}$ & $3.50 \pm 0.12^{i}$ & $3.40 \pm 0.10^{i}$ & $3.13 \pm 0.11^{j}$ & $3.37 \pm 0.13^{j}$ & $3.32 \pm 0.14^{h}$ & $3.23 \pm 0.13^{f}$ & $3.50 \pm 0.14^{j}$ \\
\hline Cinnamomum verum & $0.48 \pm 0.022^{b}$ & $0.40 \pm 0.015^{b}$ & $0.46 \pm 0.016^{b}$ & $0.32 \pm 0.013^{b}$ & $0.54 \pm 0.018^{c}$ & $0.32 \pm 0.009^{b}$ & $0.53 \pm 0.017^{c}$ & $0.38 \pm 0.012^{b}$ \\
\hline Eucalyptus globulus & $3.26 \pm 0.13^{h}$ & $3.35 \pm 0.22^{h}$ & $3.10 \pm 0.14^{\mathrm{h}}$ & $2.47 \pm 0.19^{i}$ & $3.24 \pm 0.14^{\mathrm{h}}$ & $3.52 \pm 0.19^{f}$ & $3.53 \pm 0.16^{i}$ & $3.38 \pm 0.10^{\mathrm{i}}$ \\
\hline Laurus nobilis & $2.46 \pm 0.16^{f}$ & $2.15 \pm 0.12^{\mathrm{e}}$ & $3.12 \pm 0.12^{h}$ & $2.35 \pm 0.18^{h}$ & $3.17 \pm 0.19^{9}$ & $3.12 \pm 0.11^{g}$ & $2.42 \pm 0.15^{h}$ & $2.33 \pm 0.12^{\mathrm{g}}$ \\
\hline Mentha spicata & $1.43 \pm 0.05^{d}$ & $2.15 \pm 0.19^{e}$ & $2.05 \pm 0.07^{e}$ & $1.45 \pm 0.09^{e}$ & $2.40 \pm 0.11^{f}$ & $1.22 \pm 0.04^{d}$ & $2.13 \pm 0.10^{e}$ & $2.03 \pm 0.15^{f}$ \\
\hline Mentha spp & $1.36 \pm 0.07^{d}$ & $2.36 \pm 0.18^{f}$ & $2.17 \pm 0.10^{f}$ & $1.35 \pm 0.08^{d}$ & $2.20 \pm 0.11^{\mathrm{e}}$ & $1.20 \pm 0.05^{d}$ & $2.31 \pm 0.12^{\mathrm{g}}$ & $1.53 \pm 0.15^{\mathrm{e}}$ \\
\hline Moringa oleífera & $2.32 \pm 0.04^{\mathrm{e}}$ & $1.47 \pm 0.11^{d}$ & $1.47 \pm 0.11^{d}$ & $1.55 \pm 0.09^{f}$ & $1.40 \pm 0.10^{d}$ & $2.30 \pm 0.09$ & $1.26 \pm 0.08^{d}$ & $2.34 \pm 0.12^{g}$ \\
\hline Origanum vulgare & $3.24 \pm 0.13^{h}$ & $2.40 \pm 0.10^{g}$ & $2.58 \pm 0.08^{g}$ & $3.13 \pm 0.12^{j}$ & $3.15 \pm 0.10^{\mathrm{g}}$ & $2.52 \pm 0.13^{f}$ & $2.29 \pm 0.09^{9}$ & $3.48 \pm 0.11^{j}$ \\
\hline Rosmarinus officinalis & $0.46 \pm 0.010^{b}$ & $0.53 \pm 0.12^{c}$ & $0.46 \pm 0.008^{b}$ & $0.22 \pm 0.004^{a}$ & $0.32 \pm 0.010^{a}$ & $0.32 \pm 0.008^{b}$ & $0.55 \pm 0.011^{c}$ & $0.55 \pm 0.012^{d}$ \\
\hline Salvia hispánica & $0.28 \pm 0.007^{a}$ & $0.32 \pm 0.018^{a}$ & $0.36 \pm 0.013^{a}$ & $0.22 \pm 0.015^{\mathrm{a}}$ & $0.40 \pm 0.016^{b}$ & $0.46 \pm 0.018^{c}$ & $0.46 \pm 0.027^{b}$ & $0.20 \pm 0.009^{a}$ \\
\hline Syzygium aromaticum & $3.46 \pm 0.14^{i}$ & $2.38 \pm 0.13^{\mathrm{fg}}$ & $2.19 \pm 0.12^{f}$ & $2.25 \pm 0.18^{g}$ & $3.27 \pm 0.17^{i}$ & $3.12 \pm 0.11^{g}$ & $2.12 \pm 0.12^{e}$ & $3.15 \pm 0.13^{h}$ \\
\hline Thymus vulgaris & $0.51 \pm 0.020^{c}$ & $0.33 \pm 0.014^{a}$ & $0.56 \pm 0.013^{c}$ & $0.42 \pm 0.011^{c}$ & $0.32 \pm 0.008^{a}$ & $0.22 \pm 0.002^{a}$ & $0.35 \pm 0.012^{a}$ & $0.45 \pm 0.013^{c}$ \\
\hline MEZCLA & $3.46 \pm 0.15^{i}$ & $3.57 \pm 0.22^{j}$ & $3.52 \pm 0.10^{j}$ & $3.33 \pm 0.10^{k}$ & $3.47 \pm 0.12^{k}$ & $3.57 \pm 0.14^{i}$ & $3.53 \pm 0.12^{i}$ & $3.55 \pm 0.11^{k}$ \\
\hline
\end{tabular}

* Diferente literal indica diferencia significativa entre columnas $(P<0.05)$. Los resultados están expresados en horas.

repelencia se puede deber a la especie de mosquito utilizado o a la composición y cantidad de compuestos químicos presentes en los aceites en comparación con los extractos hidro-etanólicos.

En otro estudio se ha reportado que los aceites de $S$. aromaticum y Eucalyptus, diluidos al $10 \%$, mostraron actividad repelente contra C. quinquefasciatus y A. aegypti (Sritabutra et al., 2013), sin embargo, la actividad repelente fue menor a la encontrada en este estudio. Lo anterior, se pudo deber a la baja concentración de los aceites utilizados en ese estudio. Por otro lado, el extracto metanólico de M. oleifera mostró actividad repelente contra Anopheles stephensi (Prabhu et al., 2011), mientras que el aceite de Eucalyptus mostró ser efectivo para repeler a lxodes ricinus por un tiempo de 80 min (Kulma et al., 2017).

En nuestro estudio, el extracto hidro-etanólico de $E$. globulus mostró ser más eficiente para repeler a R. microplus. Lo anterior siguiere que la tolerancia a los compuestos químicos de una planta puede variar entre las diferentes especies de garrapatas. Finalmente, Rejitha et al. (2014) reportaron que $A$. indica fue eficiente para repeler a P. americana, mostrando resultados similares a los encontrados en este estudio. Algunos autores han mencionado que los vapores de los compuestos volátiles de los alcaloides, flavonoides, terpenoides, amidas, entre otros, están asociados a la actividad repelente debido a que son moléculas tóxicas para los insectos (Vivanco et al., 2005; Celis et al., 2008). Aunque el mecanismo de acción repelente de los compuestos químicos derivados de las plantas es aún desconocido, se menciona que algunos compuestos fitoquímicos como el eucaliptol o el linalool pueden desactivar los receptores olfatorios de los insectos (Maia y Moore, 2011).

\section{CONCLUSIONES}

La evidencia indica que los extractos hidro-etanólicos de las plantas comestibles presentaron actividad antimicrobiana, insecticida y repelente, sin embargo, esta actividad biológica varió de una especie de planta a otra y su efecto no se presentó en todas las bacterias ni en todos los insectos probados cuando fueron evaluados individualmente. A pesar de lo anterior, la mezcla de extractos hidro-etanólicos aumentó significativamente la actividad antimicrobiana, insecticida y repelente; y fue efectiva contra todas las bacterias e insectos evaluados. Lo anterior sugiere que la mezcla de las fracciones solubles de estas plantas, tienen potencial como agentes antimicrobianos, insecticidas y repelentes. Por lo anterior, recomendamos realizar pruebas de campo antes de utilizar los extractos de estas plantas para el control de bacterias patógenas e insectos no deseables en la industria pecuaria; además, es importante la identificación y purificación de los compuestos químicos presentes en estas plantas para evaluar su posible mecanismo de acción antimicrobiano, insecticida y repelente.

\section{REFERENCIAS}

Abachi, S., Lee, S.y Rupasinghe, H.P. 2016. Molecularmechanisms of inhibition of Streptococcus species by phytochemicals. Molecules. 21(2): 1-31. 
Abakpa, G. O., Umoh, V. J. y Ameh, J. B. 2013. Occurrence of Vibrio cholerae in some households engaged in livestock farming in some parts of Zaria, Nigeria. Advances in Microbiology. 3(1): 128-131.

Abdullah, R. B., Embong, W. W. y Soh, H. H. 2011. Biotechnology in animal production in developing countries. 2nd International Conference on Agricultural and Animal Science. 22: 88-91.

Abraham, H. y Pal, S. K. 2014. Animal biotechnology options in improving livestock production in the horn of Africa. International Journal of Interdisciplinary and Multidisciplinary Studies. 1(3): 1-8.

Ajiboye, T. O., Mohammed, A. O., Bello, S. A., Yusuf, I. I., Ibitoye, O. B., Muritala, H. F. y Onajobi, I. B. 2016. Antibacterial activity of Syzygium aromaticum seed: Studies on oxidative stress biomarkers and membrane permeability. Microbial Pathogenesis. 95: 208-215.

Al-Mariri, A. y Safi, M. 2014. In vitro antibacterial activity of several plant extracts and oils against some gram-negative bacteria. Iranian Journal of Medical Sciences. 39(1): 36-43.

Alzamora, L., Morales, L., Armas, L. y Fernández, G. 2001. Medicina tradicional en el Perú: Actividad antimicrobiana in vitro de los aceites esenciales extraídos de algunas plantas aromáticas. Anales de la Facultad de Medicina. 62(2): 156161.

Andrade-Bustamante, G., García-López, M. A., Cervantes-Díaz, L., Aíl-Catzim, C. E., Borboa-Flores, J. y Rueda-Puente, E. O. 2017. Estudio del potencial biocontrolador de las plantas autóctonas de la zona árida del noroeste de México: Control de fitopatógenos. Revista de la Facultad de Ciencias Agrarias. Universidad Nacional de Cuyo. 49(1): 127-142.

Anyanwu, M. U. y Okoye, R. C. 2017. Antimicrobial activity of Nigerian medicinal plants. Journal of Intercultural Ethnopharmacology. 6(2): 240-259.

Ashfaq, M. y Ashfaq, U. 2012. Evaluation of mosquitocidal activity of water extract of Moringa oleifera seeds against Culex quinquefasciatus (Diptera: Culicidae) in Pakistan. Pakistan Entomologist. 34(1): 21-26.

Azwanida, N. N. 2015. A review on the extraction methods use in medicinal plants, principle, strength and limitation. Medicinal y Aromatic Plants. 4(196): 2167-0412.

Barriere, S. L. 2015. Clinical, economic and societal impact of antibiotic resistance. Journal Expert Opinion on Pharmacotherapy. 16(2): 151-153.

Borges, L. M. F., Sousa, L. A. D. D. y Barbosa, C. D. S. 2011. Perspectives for the use of plant extracts to control the cattle tick Rhipicephalus (Boophilus) microplus. Revista Brasileira de Parasitologia Veterinária. 20(2): 89-96.

Castillo, R. M., Stashenko, E. y Duque, J. E. 2017. Insecticidal and repellent activity of several plant-derived essential oils against Aedes aegypti. Journal of the American Mosquito Control Association. 33(1): 25-35.

Celis, Á., Mendoza, C., Pachón, M., Cardona, J., Delgado, W. y Cuca, L. E. 2008. Extractos vegetales utilizados como biocontroladores con énfasis en la familia Piperaceae. Una revisión. Agronomía Colombiana. 26(1): 97-106.

del Puerto Rodríguez, A. M., Suárez Tamayo, S. y Palacio Estrada, D. E. 2014. Efectos de los plaguicidas sobre el ambiente y la salud. Revista Cubana de Higiene y Epidemiología. 52(3): 372-387.
Díaz, S. M., Lugo, M. Y., Fonte, C. L., Castro, C. I., López-Vigoa, O. y Montejo, S. I. L. 2017. Evaluación de la actividad antimicrobiana de extractos frescos de hojas de Morus alba L. Pastos y Forrajes. 40(1): 43-48.

Elisha, I. L., Botha, F. S., McGaw, L. J. y Eloff, J. N. 2017. The antibacterial activity of extracts of nine plant species with good activity against Escherichia coli against five other bacteria and cytotoxicity of extracts. Complementary and Alternative Medicine. 17(133): 1-10.

Gemeda, N., Mokonnen, W., Lemma, H., Tadele, A., Urga, K., Addis, G., Debella, A., Getachew, M., Teka, F., Yirsaw, K., Mudie, K. y Gebre, S. 2014. Insecticidal activity of some traditionally used Ethiopian medicinal plants against sheep ked Melophagus ovinus. Journal of Parasitology Research. 1: $1-7$.

George, D. R., Finn, R. D., Graham, K. M. y Sparagano, O. A. 2014. Present and future potential of plant-derived products to control arthropods of veterinary and medical significance. Parasites y Vectors. 7(28): 1-12.

Jaramillo-Colorado, B. E., Duarte-Restrepo, E. y Pino-Benítez, N. 2015. Evaluación de la actividad repelente de aceites esenciales de plantas Piperáceas del departamento de Chocó, Colombia. Revista de Toxicología. 32(2): 112-116.

Kulma, M., Bubová, T., Kopecký, O. y Rettich, F. 2017. Lavender, eucalyptus, and orange essential oils as repellents against Ixodes ricinus females. Scientia Agriculturae Bohemica. 48(2): 76-81.

Kumar, P., Mishra, S., Malik, A. y Satya, S. 2012. Compositional analysis and insecticidal activity of Eucalyptus globulus (family: Myrtaceae) essential oil against housefly (Musca domestica). Acta Tropica. 122(2): 212-218.

Landers, T. F., Cohen, B., Wittum, T. E. y Larson, E. L. 2012. A review of antibiotic use in food animals: perspective, policy, and potential. Public Health Reports. 127(1): 4-22.

Maia, M. F. y Moore, S. J. 2011. Plant-based insect repellents: a review of their efficacy, development and testing. Malaria Journal. 10(1): S11.

Mandal, S. 2011. Repellent activity of Eucalyptus and Azadirachta indica seed oil against the filarial mosquito Culex quinquefasciatus Say (Diptera: Culicidae) in India. Asian Pacific Journal of Tropical Biomedicine. 1(1): 109-112.

Manyi-Loh, C. E., Mamphweli, S. N., Meyer, E. L., Makaka, G., Simon, M. y Okoh, A. I. 2016. An overview of the control of bacterial pathogens in cattle manure. International Journal of Environmental Research and Public Health. 13(843): 1-27.

Martínez, I. y Cruz, M. 2009. El uso de químicos veterinarios y agrícolas en la zona ganadera de Xico, centro de Veracruz, México, y el posible impacto ambiental. Acta Zoológica Mexicana. 25(3): 673-681.

Monteiro, I. N., dos Santos Monteiro, O., Costa-Junior, L. M., da Silva Lima, A., de Aguiar Andrade, E. H., Maia, J. G. S. y Mouchrek Filho, V. E. 2017. Chemical composition and acaricide activity of an essential oil from a rare chemotype of Cinnamomum verum Presl on Rhipicephalus microplus (Acari: Ixodidae). Veterinary Parasitology. 238: 54-57.

Mostafa, A. A., Al-Askar, A. A., Almaary, K. S., Dawoud, T. M., Sholkamy, E. N. y Bakri, M. M. 2017. Antimicrobial activity of some plant extracts against bacterial strains causing food poisoning diseases. Saudi Journal of Biological Sciences. https://doi.org/10.1016/j.sjbs.2017.02.004. 
Munuswamy, H., Thirunavukkarasu, T., Rajamani, S., Elumalai, E. K. y Ernest, D. 2013. A review on antimicrobial efficacy of some traditional medicinal plants in Tamilnadu. Journal of Acute Disease. 2(2): 99-105.

Niroumand, M. C., Farzaei, M. H., Razkenari, E. K., Amin, G., Khanavi, M., Akbarzadeh, T. y Shams-Ardekani, M. R. 2016. An evidence-based review on medicinal plants used as insecticide and insect repellent in traditional iranian medicine. Iranian Red Crescent Medical Journal. 18(2): 1-8.

Panchal, P., Bajaj, H. y Maheshwari, S. 2013. Azadirachta indica (NEEM): Antibacterial effects against Escherichia coli and Salmonella. Guru Drone Journal of Pharmacy and Research. 1(1): 18-21.

Pimentel, D. y Burgess, M. 2014. Environmental and economic costs of the application of pesticides primarily in the United States. En: Integrated Pest Management, pp. 47-71. Springer Netherlands.

Prabhu, K., Murugan, K., Nareshkumar, A., Ramasubramanian, N. y Bragadeeswaran, S. 2011. Larvicidal and repellent potential of Moringa oleifera against malarial vector, Anopheles stephensi Liston (Insecta: Diptera: Culicidae). Asian Pacific Journal of Tropical Biomedicine. 1(2): 124-129.

Pulido, S. N. J. y Cruz, C. A. 2013. Eficacia de los extractos hidroalcohólicos de dos plantas sobre garrapatas adultas Rhipicephalus (Boophilus) microplus. Corpoica Ciencia y Tecnología Agropecuaria. 14(1): 91-97.

Rejitha, T. P., Reshma, J. K. y Mathew, A. 2014. Study of repellent activity of different plant powders against cockroach (Periplanata americana). International Journal of Pure and Applied Bioscience. 2(6), 185-194.

Rodríguez-Vivas, R. I., Rosado-Aguilar, J. A., Ojeda-Chi, M. M., Pérez-Cogollo, L. C., Trinidad-Martínez, I. y Bolio-González, M. E. 2014. Control integrado de garrapatas en la ganadería bovina. Ecosistemas y Recursos Agropecuarios. 1(3): 295308.

Rodríguez-Vivas, R. I., Grisi, L., Pérez de León, A. A., Silva, V. H., Torres-Acosta, J. F. J., Fragoso, S. H., Romero, S. D., Rosario, C. R., Saldierna, F. y García, C. D. 2017. Evaluación del impacto económico potencial de los parásitos del ganado bovino en México. Revisión. Revista Mexicana de Ciencias Pecuarias. 8(1): 61-74.

Sebei, K., Sakouhi, F., Herchi, W., Khouja, M. L. y Boukhchina, S. 2015. Chemical composition and antibacterial activities of seven Eucalyptus species essential oils leaves. Biological Research. 48(7): 1-5.
Simonetti, A. P. M. M. y Christ, D. 2017. Hydroalcoholic extract of crambe on Sitophilus zeamais insects and maize seed quality. Journal of Agricultural Science. 10(1): 283-292.

Shaheen, M., Tantary, H. A. y Nabi, S. U. 2016. A treatise on bovine mastitis: Disease and disease economics, etiological basis, risk factors, impact on human health, therapeutic management, prevention and control strategy. Advances in Dairy Research. 4(1): 1-10.

Silva, N. C .C. y Fernandes, J. A. 2010. Biological properties of medicinal plants: a review of their antimicrobial activity. Journal of Venomous Animals and Toxins Including Tropical Diseases. 16(3): 402-413.

Sritabutra, D. y Soonwera, M. 2013. Repellent activity of herbal essential oils against Aedes aegypti (Linn.) and Culex quinquefasciatus (Say.). Asian Pacific Journal of Tropical Disease. 3(4): 271-276.

Tindo, D. S., Amusant, N., Dhangou, J., Watu, D. V., Avlessi, F., Dahouenon, A. E., Lozano, P., Pioch, D. y Sohounihloue, K. C. D. 2012. Screening of repellent, termiticidal and preventive activities on wood, of Azadirachta indica and Carapa procera (Meliaceae) seeds oils. Journal of Biological Sciences. 1(3): 25-29.

Vázquez-Luna, A., Pérez-Flores, L. y Díaz-Sobac, R. 2007. Biomoléculas con actividad insecticida: Una alternativa para mejorar la seguridad alimentaria. CYTA-Journal of Food. 5(4): 306-313.

Vivanco, J. M., Cosio, E., Loyola-Vargas, V. M. y Flores, H. E. 2005. Mecanismos químicos de defensa en las plantas. Investigación y Ciencia. 341(2): 68-75.

Volpato, A., Baretta, D., Zortéa, T., Campigotto, G., Galli, G. M., Glombowsky, P., Santos, R. C. V., Quatrin, P. M., Ourique, A. F., Daldissera, M. D., Stefani, L. M. y Da Silva, A. S. 2016. Larvicidal and insecticidal effect of Cinnamomum zeylanicum oil (pure and nanostructured) against mealworm (Alphitobius diaperinus) and its possible environmental effects. Journal of Asia-Pacific Entomology. 19(4): 1159-1165.

War, A. R., Paulraj, M. G., Ahmad, T., Buhroo, A. A., Hussain, B., Ignacimuthu, S. y Sharma, H. C. 2012. Mechanisms of plant defense against insect herbivores. Plant Signaling y Behavior. 7(10): 1306-1320.

Zoubiri, S. y Baaliouamer, A. 2014. Potentiality of plants as source of insecticide principles. Journal of Saudi Chemical Society. 18(6): 925-938. 